\title{
Performance Measurement for People with Multimorbidity and Complex Health Needs
}

Walter P. Wodchis

\begin{abstract}
This paper reviews approaches to performance measurement in health systems with particular attention to people with multimorbidity and complex health needs. Performance measurement should be informative and used by multiple stakeholders in order to align performance improvement efforts. System performance measures must allow for macrosystem and meso-organization and provider-level reporting, and they should be relevant and important to stakeholders at each level, as well as to patients and all potential care recipients. Measures that assess health outcomes and individuals' experiences with providers, including care planning and coordination of care across providers, are essential to assess value for people with multimorbidity and complex health needs. I suggest that performance measurement for this population should be motivated by the Complexity Framework and organized by the Triple Aim. Based on the care needs and appropriate goals for the health system for this population, applicable measures and suggestions for implementing and using performance measurement systems are identified. Particularly in the case of people with multimorbidity and complex health needs, performance measures must move beyond measures specific to individual encounters to track care for people over time and space. Measures must be rooted in individuals' own needs and goals for care. New systems are required to enable collection and reporting of these measures.
\end{abstract}

\section{Introduction}

Measuring and reporting on the performance of the health system allows patients, carers, providers and health system decision-makers to identify whether the health system is doing what it should and if patient and population health outcomes are optimized. In conjunction with goals or benchmarks, performance measurement also allows stakeholders to be aware of performance gaps and opportunities for improvement. Performance measures that closely reflect the goals established for the health system are necessary to assess progress.

Health system performance measures should serve to measure the value of health produced from the delivery of health and social services. Performance measurement should be informative and used by multiple stakeholders in order to align performance improvement efforts. The assessment of provider effectiveness and quality, constituent resources and associated costs, as well as individuals' experiences and outcomes are all important goals of health system performance.

System performance measures must allow for macro-system and meso-organization and provider-level reporting, and they should be relevant and important to stakeholders at each level, as well as to patients and all potential care recipients. General population health measures of life expectancy, health-related quality of life and well-being that are relevant to the entire population should be included, alongside specific measures for particular populations such as the effective coordination of care among multiple providers for people with complex health needs. Performance measures 
should reflect the goals of providers and patients, and resolve differences when these are not aligned (Kuluski et al. 2013).

This monograph begins with an overview of the dominant approaches to performance measurement in health systems across industrialized nations with particular relevance to Canada. Because the focus here is performance measurement for people with multimorbidity and complex health needs, a brief review of the care needs and appropriate goals for the health system for this population is necessary. I then turn to applicable measures and end with some suggestions for implementing and using performance measurement systems. Particularly in the case of people with multimorbidity and complex health needs, performance measures must move beyond measures specific to individual encounters to track care for people over time and space.

\section{Current State of Performance Measurement}

Performance measurement in healthcare has developed into a major activity for payers, regulators and providers. For decades, international comparisons of health system performance have reported health outcomes such as infant mortality, overall life expectancy and, more recently, disability-adjusted life expectancy or equivalent measures. The Organization for Economic Co-operation and Development, the World Health Organization and the Institute of Medicine, have developed comprehensive approaches to performance measurement that examine dimensions such as the accessibility, safety and effectiveness of healthcare services in addition to health outcomes.

Within health systems, a performance framework often determines the focus and selection of performance measures for reporting. Common frameworks applied to health service providers within health systems include Kaplan and Norton's balanced scorecard (BSC) (Kaplan and Norton 1992) and Donabedian's structure, process and outcome (SPO) frameworks (Donabedian 1966). While the BSC approach can be used in comparative analyses to identify areas for improvement, it fails to explicitly clarify the contributory relationships between dimensions of performance and overall health outcomes. The SPO approach, on the other hand, can be most clearly specified when applied to a specific production process for a defined care pathway but may be more difficult to use at a broad system level when many resources are simultaneously engaged in a wide variety of activities.

The more recent Triple Aim framework promoted by the Institute for Healthcare Improvement (Berwick et al. 2008) focuses on health experience and outcomes while considering costs (representing resources). Unlike other health system performance approaches, the Triple Aim does not explicitly specify attributes such as accessibility or safety. In comparison with the BSC and SPO frameworks, the Triple Aim also does not explicitly designate structural or organization-level measures. Rather than focusing on these activities, it suggests measuring the contribution of provider activities towards achievement of cost, experience and health outcomes. It does however provide a more explicit assessment of value. Central to the Triple Aim is the identification of a defined population and emphasis on patient experience inclusive of patient engagement, continuity, safety and evidence-based clinical practices (Berwick et al. 2008).

Increasingly, health systems are adopting the Triple Aim framework and implementing more robust measurement of patient experience. Ensuring that the patient's perspective is included within a performance measurement system is the only way to evaluate progress towards providing truly patient-centred care (Cuthbertson 2015). Both patient-reported outcome measures (PROMs) and patient-reported experience measures (PREMs) provide a mechanism to include patients' perspectives. However, the variability in patient care needs provides a challenge for the use and selection of standard measures (Miller et al. 2015). Most examples of PREMs and PROMs are based on specific encounters or episodes of care. However, outcomes and experience among individuals with multiple complex health needs are dependent upon the contributions from many different providers over extended periods. Generic health status measures are possible PROMs for individuals with complex health needs. Patient experience measurement must enable patients who receive care from multiple providers to report on their experience from many of these providers (Cuthbertson 2015). Experience across encounters can be tied together to assess the degree of coordination and integration across providers. In Ontario, The Change Foundation's Partners Advancing Transitions in Healthcare (PATH) project provided an opportunity for patients to report regularly on their experiences with various encounters and provide summative experiences of the degree of coordination across care providers (The Change Foundation 2016). But patients were not themselves directly consulted in determining what measures they would report on. If the aim of the measurement is to provide a patient-centred outcome, then patients themselves should be involved in the development of the measurement instruments (Trujols et al. 2013). Steele Gray and colleagues found that engaging with patients in designing measurement lead to a vastly different approach to measurement, focusing on individual goals and goal attainment (Steele Gray et al. 2016a).

\section{What Characterizes Good Care for People with Complex Health Needs?}

Accepting that health system performance measurement is important and that measures need to engage and be meaningful to people and providers, I turn now to consider what needs to be measured. To evaluate the Triple Aims of health, experience and cost for people with complex health needs, we will need a broad perspective. The health for this population is determined by a multiplicity of conditions and interventions. Many different providers are involved over time, and both medical and social care programs may be instrumental in achieving 
health. An assessment of performance must be reflective of whether needs are being met and whether aims and goals are being achieved.

The Complexity Framework developed by Schaink and colleagues (2012) recognizes medical and physical health, mental health, demographics, health and social experiences and social capital as contributing factors to health among individuals with complex health needs. There are other factors, but these capture the essence that it is multifaceted. The complexity framework highlights that while some needs are clearly medical, there are many areas of need that are entirely outside of how most industrialized nations have implemented medical care. Within an individual with multimorbidity and complex health needs, these are not independent or isolated factors. There is clear interplay and interdependency among them. A heart failure patient without a scale will find it difficult to self-manage fluid retention and weight. A diabetic without enough money for healthy food will find it difficult to manage their diet. An adult with strong social capital, high education and adequate economic resources is likely to manage far better than a disenfranchised and isolated individual in the face of similar medical conditions. These are individual needs and they speak to the variability in individual complexity. Consequently, this conceptualization also highlights the challenge of having performance measures that are relevant to individuals with different care needs and are applicable across individuals with a wide variety of health and social circumstances. A system of measurement must be specific to individuals but still allow for a common approach to evaluating outcomes and aggregating these outcomes to provider and system levels.

Health outcomes for people with complex health needs must reflect overall quality of life resulting from having multiple needs met. Individual's experience must capture dimensions of importance to people and providers. Focus groups with people with complex chronic disease and disability and their caregivers revealed specific needs including strong communication and dialogue between themselves and their providers, rapid access to appointments, transportation and cost (Steele Gray et al. 2014). Individuals must be respectfully engaged in planning and feel confident about managing their health as a result. Care must be delivered by a coordinated interdisciplinary team effort inclusive of all health and social services. In this regard, providers' aims and contributions must also be considered. Good clinical care and an absence of conflicting treatments, such as from polypharmacy, are relevant and under the control of health providers. The economic goal is to ensure that resources are efficiently deployed to maximize health and experience. Resources include public spending for healthcare and social care and individuals' own costs for obtaining health-producing services. Performance measurement for people with complex health needs must capture the summative experience and health outcomes across services that are needed to support this population.

\section{Performance Measures for People with Multimorbidity and Complex Health Needs}

The health system should improve patient and population health. It should address the needs of the population. It must also consider differential needs and goals among subgroups of the population. The majority of the population uses very few services, and their needs can be met with availability of primary care. In contrast, individuals with multimorbidity and complex health needs are met with a multiplicity of services and interventions. Because integrated care programs are the most appropriate organizational approach, recommended measures for such programs are a useful basis for departure (e.g., Palmer et al. 2006). The US National Quality Forum has gathered extensive information on management and measurement for people with multiple chronic conditions. It recommends the use of outcome measures when available and process measures that are most closely linked to outcomes otherwise (National Quality Forum 2012).

Organizing recommended measures along the Triple Aim framework enables measurement to be goal-oriented. Measures of health, experience and cost aims are needed. Measurement should encompass objective and subjective approaches within each aim. The core set of measures should apply to all of the population. In this regard, disease- or condition-specific measures that may be important for some (e.g., diabetes care measures) may not be suitable for people with complex health needs unless a vast majority of the population has the same clinical needs and desired outcomes for such measures. Table 1 suggests some sample measures of performance applicable to people with multimorbidity and complex health needs. It includes objective measures for health (e.g., potential years of life lost), experience (e.g., wait times for services) and cost. It also suggests subjective measures, including PROMs and PREMs and patient-reported costs.

TABLE 1.

Example performance measures for people with multimorbidity and complex health needs

\begin{tabular}{|c|c|c|}
\hline Aim & Objective measures & Subjective measures \\
\hline \multirow[t]{2}{*}{ Health } & $\begin{array}{l}\text { - Potential years of life lost } \\
\text { - Life expectancy }\end{array}$ & $\begin{array}{l}\text { - Self-reported health } \\
\text { - EQ-5D, PROMIS or VR-12 }\end{array}$ \\
\hline & \multicolumn{2}{|c|}{$\begin{array}{l}\text { Disability-adjusted life years can incorporate both subjective } \\
\text { health and objective measures of life expectancy }\end{array}$} \\
\hline Experience & $\begin{array}{l}\text { - Wait time for consultation } \\
\text { or other service }\end{array}$ & $\begin{array}{l}\text { - Continuity of care } \\
\text { - Involvement in care } \\
\text { - Coordination of care } \\
\text { - Self-activation } \\
\text { - Unmet needs (access) } \\
\text { - Caregiver experience }\end{array}$ \\
\hline Cost & $\begin{array}{l}\text { - Health system cost } \\
\text { - Social service costs }\end{array}$ & $\begin{array}{l}\text { - Individual and carer } \\
\text { opportunity cost, including } \\
\text { financial and non-financial }\end{array}$ \\
\hline
\end{tabular}

EQ-5D = Euro00L group Health-Related Quality of Life measure; PROMIS = Patient-Reported Outcomes Measurement Information System; VR-12 = Veterans RAND 12-item survey. 
In addition to life expectancy or potential years of life lost measures, disability-adjusted life years can be measured by combining life-expectancy with a preference- or utility-based measure that assesses health attainment. The EuroQOL-5 Dimension (EQ-5D) Survey is a utility-based measure widely used in Europe that captures health status while the Veterans RAND 12 Item Health Survey (VR-12) captures individual's capability in usual activities and roles; either would be appropriate for measuring individual and population health status.

\section{The focus on people with complex health needs is relatively recent, and health systems have generally not fully addressed the needs of this population.}

The National Institute of Medicine's PROMIS measures can also be suitable, though not all have preference-based measurement scales. From a subjective perspective, the simple question of health status on a scale from poor to excellent should not be overlooked. Measures that assess experiences with individual providers, including care planning and coordination of care across providers, are essential to assess value for people with complex health needs. Using composite measures that reflect care over time and across multiple providers can enable shared accountability among providers while still allowing constituent factors to be reported. Measuring cost to the payer and also to the individual patient and their caregivers is required to assess value in relation to the economic cost. Additional measurement of caregiver experiences is important to understand the contributory relationships between these factors and performance. This approach to measurement aligns with the Triple Aim goals of population health, patient experience and cost and can provide a strong foundation for health system performance measurement.

Table 1 is not intended to represent a complete set of measures. In addition to standardized PROMs, novel patientdefined outcomes are an important basis for measurement. Defining goals at the individual level through a discussion between individuals and their health providers and measuring achievement of goals using Goal Attainment Scaling techniques allow for an individualized and comparable approach to performance measurement (Steele Gray et al. 2016b). Measuring the extent of provider coordination of care that patients experience is important, but the provider perspective on this measure may also be important. Some have argued that provider-level measurement is an important gap in the Triple Aim framework (Bodenheimer and Sinsky 2014). Providers themselves can provide an important assessment of team functioning through a measure such as the Team Climate Inventory (Anderson and West 1998). Provider experience and engagement in their organizations may also be an important measure of the care that individuals experience as patients.

\section{The Way Forward}

Participants need to be involved in assessing the value created by the health system. This is particularly important for people with multimorbidity and complex health needs. We already have well-established measurement systems for population health outcomes. However, these data need to be harvested and reported at the individual, provider and local system levels. We lack rigorous implementation of patient experience measures, particularly for individuals with complex health needs who have many encounters with many providers to meet their care needs. We need to innovate performance measurement to be more person-centred.

The focus on people with complex health needs is relatively recent, and health systems have generally not fully addressed the needs of this population. Some more mature programs, such as the Program of All-Inclusive Care for the Elderly (PACE) in the United States, might offer some insight. PACE is one of the largest programs, and evaluations have assessed its value. PACE programs are required to track enrolments, annual assessments of physical and mental health, mortality, a few clinical indicators (falls, pressure ulcers) and a sample of participants' report on satisfaction and health-related quality of life using the VR-12 as well as self-reported activities of daily living (CMS 2011). However, the use of these measures in performance reporting to participants and providers is unknown. There are also many gaps relative to recommended measures for integrated care programs (Palmer et al. 2006).

Most promising programs are still in pilot, developmental or research stages. The PATH project in Ontario provided useful weekly feedback to providers regarding patient's experiences but participants did not engage fully in reporting (The Change Foundation 2016). The TAPESTRY program, also in Ontario, includes a patient-oriented approach to setting goals and sharing information. But the use of information collected for performance measurement purposes is not an apparent fixture of the program (http://www.healthtapestry.ca/ accessed May 16, 2016). An upcoming trial of patient-centred goal-setting, goal-attainment measurement and health outcomes is similarly still experimental (Steele Gray 2016b).

While quality improvement and performance measurement are not the same in purpose and measurement and reporting needs differ, there is considerable overlap, and implementing a measurement system as part of care delivery can support measurement for both activities. Such alignment is necessary to be relevant to providers and the people that they care for. Although such systems are not currently widespread, they should be built-in to newly emerging patient-accessed health records systems. Payers are in a position to support the implementation and meaningful use of such systems, similar to the implementation of the Health Information Technology for Economic and Clinical Health (HITECH) Act in the US (Blumenthal and Tavenner 2010). Implementation should enable 
patients, providers, payers and regulators to have access to performance results in order to guide system management and researchers to develop new knowledge and guide system improvement.

Measuring the value created by health services requires measurement of individual and population health outcomes, individual experience of care and the economic costs associated with health outcomes. For individuals with multimorbidity and complex health needs, assessing value requires innovations in measurement and reporting. Arguably, the patient experience should serve as the foundation for health system performance measurement, but, unequivocally, patient-determined outcomes are the most relevant outcomes for people with multimorbidity and complex health needs. Measurement that engages providers to optimize outcomes for people whose care they support will result in better value from the health system. HQ

\section{References}

Anderson, N.R. and M.A. West. 1998. "Measuring Climate for Work Group Innovation: Development and Validation of the Team Climate Inventory." Journal of Organizational Behavior 19: 235-58.

Berwick, D.M., T.W. Nolan and J. Whittington. 2008. "The Triple Aim: Care, Health, and Cost." Health Affairs (Millwood) 27(3): 759-69. doi:10.1377/hlthaff.27.3.759.

Blumenthal, D. and M. Tavenner. 2010. "The "Meaningful Use" Regulation for Electronic Health Records." New England Journal of Medicine 363: 501-04.

Bodenheimer, T. and C. Sinsky. 2014. "From Triple to Quadruple Aim: Care of the Patient Requires Care of the Provider." Annals of Family Medicine 12(6): 573-76.

CMS - Centres for Medicare and Medicaid Services. 2011. "Programs of All-Inclusive Care for the Elderly (PACE)Manual. Chapter 10: Quality Assessment and Performance Improvement." CMS Manual 100-11. Rev 2. Retrieved February 10, 2016. <https://www.cms. gov/Regulations-and-Guidance/Guidance/Manuals/Internet-OnlyManuals-IOMs-Items/CMS019036.html>.

Cuthbertson, L. 2015. "Patient-Centred Measurement in British Columbia: Statistics without the Tears Wiped Off." HealthcarePapers 14(4): 46-54.

Donabedian, A. 1966. "Evaluating the Quality of Medical Care." Millbank Memorial Fund Quarterly 44(3): 691-729.

Kaplan, R. and D. Norton. 1992. "The Balanced Scorecard: Measures That Drive Performance." Harvard Business Review 70(1): 71-79.

Kuluski, K., A. Gill, G. Naganathan, R. Upshur, R.L. Jaakkimainen and W.P. Wodchis. 2013. "A Qualitative Descriptive Study on the Alignment of Care Goals Between Older Persons with MultiMorbidities, their Family Physicians and Informal Caregivers." BMC Family Practice 14: 133. doi:10.1186/1471-2296-14-133.
Miller, D., C. Steele Gray, K. Kuluski and C. Cott. 2015. "PatientCentered Care and Patient-Reported Measures: Let's Look Before We Leap." Patient - Patient-Centered Outcomes Research 8: 293-99.

National Quality Forum. 2012. "MCC Measurement Framework." Retrieved April 10, 2016. <http://www.qualityforum.org/Projects/ Multiple_Chronic_Conditions_Measurement_Framework.aspx>.

Schaink, A.K., K. Kuluski, R.F. Lyons, M. Fortin, A.R. Jadad, R. Upshur et al. 2012. "A Scoping Review and Thematic Classification of Patient Complexity: Offering a Unifying Framework." Journal of Comorbidity 2(1): 1-9.

Palmer, L., K.L. Lanos, C. Tobias and M. Bella. 2006. Integrated Care Program: Performance Measures Recommendations. Center for Health Care Strategies. Retrieved June 27, 2016. <http://www. chcs.org/resource/integrated-care-program-performance-measuresrecommendations/>.

Steele Gray, C., D. Miller, K. Kuluski and C. Cott. 2014. "Tying eHealth Tools to Patient Needs: Exploring the Use of eHealth for Community-Dwelling Patients with Complex Chronic Disease and Disability." JMIR Research Protocols 3(4): e67.

Steele Gray, C, A.I. Khan, K. Kuluski, I. McKillop, S. Sharpe, A.S. Bierman et al. 2016a. "Improving Patient Experience and Primary Care Quality for Patients with Complex Chronic Disease Using the Electronic Patient-Reported Outcomes Tool: Adopting Qualitative Methods into a User-Centered Design Approach." JMIR Research Protocols 5(1): e28.

Steele Gray, C., W.P. Wodchis, R. Upshur, C. Cott, B. McKinstry, B. Mercer, et al. 2016b. "Supporting Goal-Oriented Primary Health Care for Seniors with Complex Care Needs Using Mobile Technology: Evaluation and Implementation of the Health System Performance Research Network, Bridgepoint Electronic Patient Reported Outcome Tool." JMIR Research Protocols 5(2):e126.

The Change Foundation. 2016. "Charting a New Path: Part 2 - Creating Meaningful Partnerships in Care: Lessons from West Northumberland." Retrieved April 10, 2016. <http://www.changefoundation.ca/site/ wp-content/uploads/PATH-report_pt2-final.pdf>.

Trujols, J., M.J. Portella, I. Iraurgi, M.J. Campins, N. Siñol and J.P.D.L. Cobos. 2013. "Patient-Reported Outcome Measures: Are They Patient-Generated, Patient-Centred or Patient-Valued?" Journal of Mental Health 22(6): 555-62.

\section{About the Author \\ Walter $\mathbf{P}$. Wodchis, $\mathrm{PhD}$, is associate professor in the Institute of Health Policy Management and Evaluation at the University of Toronto, ON. He is also a research scientist at the Toronto Rehabilitation Institute and adjunct scientist at the Institute for Clinical Evaluative Sciences.}

\section{HealthcareQuarterly.com}

